\title{
Mapeamento da produção científica sobre o Programa Mais Médicos
}

\section{Mapping of scientific production on the Mais Médicos Program}

\author{
David Ramos da Silva Rios ${ }^{a}$ \\ aniversidade Federal da Bahia. Faculdade de Medicina. \\ Salvador, BA, Brasil. \\ E-mail: david-riosळhotmail.com \\ Carmen Teixeirab \\ bUniversidade Federal da Bahia. Instituto de Humanidades, \\ Artes e Ciências. Salvador, BA, Brasil. \\ E-mail: carmentळufba.br
}

\section{Resumo}

O Programa Mais Médicos (PMM) foi instituído pela Lei $\mathrm{n}^{0} 12.871$, de 22 de outubro de 2013 , com a finalidade de reduzir as desigualdades regionais na área da saúde e garantir acesso igualitário e integral aos serviços de saúde. A implantação desse programa tem gerado interesse nos pesquisadores da área de política, planejamento e gestão em saúde, interessados em analisar seu processo de formulação e implementação, bem com os resultados alcançados até o momento. O objetivo deste trabalho é efetuar uma revisão integrativa da literatura sobre o PMM em diferentes bases de dados - Biblioteca Virtual em Saúde, portal de periódicos da Coordenação de Aperfeiçoamento de Pessoal de Nível Superior (Capes) e plataforma de pesquisa do Programa Mais Médicos -, visando identificar seus principais temas de investigação, os pesquisadores e os resultados de estudos. Foram identificados 137 trabalhos, publicados entre os anos de 2013 a 2016, classificados segundo o ano de publicação, o tipo de trabalho, o idioma, a área do conhecimento, temas e subtemas abordados, a procedência institucional dos autores e os resultados apresentados. A maioria dos estudos procede da área centro-sul do Brasil, sendo pesquisas empíricas que analisam ou avaliam o programa e apontam que o PMM tem reduzido a escassez de médicos em determinadas regiões do país, melhorado a relação médico/paciente, estimulado práticas interdisciplinares e fortalecido a atenção primária no Sistema Único de Saúde (SUS). Indicam, também, alguns limites do PMM, notadamente a inadequação do perfil dos profissionais que dele participam, o que demanda o fortalecimento das iniciativas de formação e educação permanente do pessoal inserido no programa.

Palavras-chave: Mais Médicos; Política de Saúde; Formação Médica. 
The Mais Médicos Program (MMP) was established by Law no. 12,871 of October, 22nd 2013 with the purpose of reducing regional inequalities in health and ensuring full and egalitarian access to health services. The implementation of this program has generated interest in health policy, planning, and management researchers, who intend to analyze its formulation and implementation process, as well as the results achieved until now. The objective of this article is to perform an integrative literature review concerning the MMP in different databases - Biblioteca Virtual em Saúde, journal platform from Higher Education Improvement Coordination (Capes) and research platform from Mais Médicos Program -, to identify the main themes of investigation, researchers, and study results. We identified 137 articles published between 2013 and 2016, which were classified according to the year of publication, type of publication, language, field of knowledge, themes and subthemes approached, author's affiliation, and results. Most studies were published on Brazil's central south region, and were empirical studies evaluating or analyzing the program, pointing that MMP has reduced the shortage of physicians in some regions of the country, improved the doctor-patient relationship, stimulated interdisciplinary practices and consolidated primary care in the Brazilian National Health System (SUS). They also indicate some of the program's limitations, especially the inadequacy of the profile of the professionals who integrate it, which demand the strengthening of the permanent educational initiatives for the personnel inserted in the program.

Keywords: Mais Médicos; Health Policy; Medical Education.
Em 2014, o Brasil vivia os preparativos para a Copa do Mundo em uma conjuntura que envolvia aumento da inflação, surgimento de denúncias importantes de corrupção e aumento dos gastos públicos com a construção de estádios e melhoria da infraestrutura nacional para o recebimento do evento esportivo. É justamente nesse contexto que surgem as chamadas "jornadas de junho" (Magno; Paim, 2015), caracterizada, pelas críticas à situação social existente no país e aos elevados gastos públicos com a Copa do Mundo e pela demanda por ampliação da oferta e melhoria da qualidade dos serviços públicos.

Dentre as reivindicações apresentadas pelos movimentos, destacam-se as relativas à ampliação e melhoria da qualidade da assistência à saúde, simbolizadas pelo slogan "Hospitais padrão Fifa", alusão ao volume de recursos dispendidos na construção das "arenas" quando comparado ao processo histórico de subfinanciamento do Sistema Único de Saúde (SUS). ${ }^{3}$

Como respostas a essas demandas, a então presidente da república, Dilma Rousseff, propôs os "Pactos em Favor do Brasil" (Bueno; Silva, 2015), que incluíram, entre suas prioridades, a intervenção em um problema que desde 2011 ocupava espaço na agenda do Governo Federal: as desigualdades na distribuição de médicos no território brasileiro e a escassez desses profissionais no país. As medidas adotadas, a exemplo do Programa de Valorização do Profissional da Atenção Básica (Provab) e o Fundo de Financiamento Estudantil (Fies), haviam se revelado insuficientes para promover a desconcentração dos médicos e sua inserção nos municípios de pequeno porte das várias regiões do país, o que favoreceu a decisão e a implantação do Programa Mais Médicos (PMM), criado em julho de 2013 por meio de uma

\footnotetext{
1 Segundo Magno e Paim (2015), o período das manifestações, no Brasil, pode ser dividido em quatro fases: lutas contra o aumento das tarifas de transporte e crítica ao movimento pela mídia brasileira; crescimento do apoio popular por meio das redes sociais e aumento das manifestações com pautas progressistas e de luta por direitos sociais; adesão em massa, com apoio e tentativa de interferência da grande mídia; e perda da força das manifestações e ampliação da participação de certos grupos, como a esquerda tradicional e os black blocs. As jornadas envolveram vozes de diferentes atores e se incluíram em uma onda de mobilizações contemporâneas, com reivindicações próprias, organização particular e criação de interação singular entre os indivíduos, o movimento e a sociedade (Bringel; Pleyers, 2013).

2 Fédération Internationale de Football Association, ou Federação Internacional de Futebol, em português.

3 Cf. Magno e Paim (2015).
} 
medida provisória posteriormente convertida em lei em outubro do mesmo ano - a Lei ${ }^{0} 12.871$, de 22 de outubro de 2013 (Brasil, 2013b).

Estruturado em três pilares (provimento emergencial de médicos, educação médica e infraestrutura), o PMM busca formar recursos humanos na área médica para atuar no SUS, orientando o funcionamento dos cursos de medicina no país e reduzindo as desigualdades regionais na área da saúde, garantindo assim acesso igualitário e integral a todos (Brasil, 2013b). A novidade apresentada pelo PMM diz respeito, portanto, à conjugação da proposta de redistribuição dos profissionais médicos com ênfase atribuída à implementação de mudanças no processo de formação médica, seja pelos programas de residência, seja pelo estímulo à introdução de inovações metodológicas nos cursos de graduação.

O programa tem oito objetivos principais: diminuir a carência de médicos nas regiões prioritárias para o SUS; fortalecer a prestação de serviços na atenção básica; aprimorar a formação médica com aumento da quantidade de campos de prática; ampliar a inserção do médico em formação nas unidades de atendimento do SUS; fortalecer a política de educação permanente com a integração educação-serviço por meio da supervisão acadêmica; promover a troca de experiências entre médicos brasileiros e estrangeiros; estimular pesquisas voltadas ao SUS; e aperfeiçoar os médicos para atuação em políticas públicas de saúde (Brasil, 2015).

Para garantir a consolidação de tais objetivos, algumas medidas vêm sendo tomadas, a exemplo da reordenação da oferta de cursos médicos em regiões prioritárias, ${ }^{4}$ do lançamento das novas Diretrizes Curriculares Nacionais (DCN) para os cursos de graduação em Medicina ${ }^{5}$ (Brasil, 2014), do aperfeiçoamento de médicos na área de Atenção Básica em Saúde, inclusive por meio de supervisões, e do provimento de médicos, mesmo estrangeiros, para aquelas regiões onde há dificuldade em garantir a fixação de profissionais brasileiros.
Após três anos de implantação, o PMM tem apresentado resultados significativos: a abertura de $\mathbf{1 8 . 2 4 0}$ postos de emprego em 4.058 municípios de todo o país, cobrindo $73 \%$ das cidades brasileiras, e 34 Distritos Sanitários Especiais Indígenas (DSEI); a autorização da criação de mais de cinco mil vagas em cursos de graduação em Medicina e quase cinco mil em cursos de Residência Médica; e a liberação de um investimento da ordem de R\$ 5 bilhões para o financiamento de melhorias nas unidades de saúde (Brasil, 2015).

Apesar desses avanços, inúmeras críticas ainda são feitas ao PMM, principalmente por parte de entidades representativas da categoria médica (Soares et al., 2017). É importante, portanto, acompanhar o desenvolvimento do programa, que pode ser feito inclusive pela análise da produção científica em torno desse tema. Assim, o objetivo deste artigo é realizar uma revisão integrativa da literatura sobre o PMM em diferentes bases de dados - Biblioteca Virtual em Saúde, portal de periódicos da Coordenação de Aperfeiçoamento de Pessoal de Nível Superior (Capes), plataforma de pesquisa do PMM - no período 2013-2016, buscando identificar os principais temas de pesquisa referentes ao programa e a procedência institucional dos pesquisadores envolvidos e analisar os resultados apresentados e publicados até então, buscando discutir a contribuição desse programa à construção do SUS e ao processo de Reforma Sanitária Brasileira (RSB).

\section{Metodologia}

Trata-se de um estudo de revisão integrativa da literatura, baseado na aplicação de métodos explícitos e sistematizados de busca, apreciação crítica e síntese da informação selecionada (OPS, 2013). O mapeamento da produção científica implicou, inicialmente, a definição do descritor de assunto, escolhido por meio da análise daqueles disponíveis em trabalhos anteriores sobre a mesma temática, escolhendo-se o descritor "Programa Mais Médicos".

4 O Ministério da Educação (MEC), após a indicação das regiões prioritárias pelo Ministério da Saúde, lança editais para a abertura de novos cursos.

5 As novas Diretrizes Curriculares Nacionais (DCN) propõem a instituição de uma carga horária mínima em Atenção Básica durante a formação médica, bem como a implementação de processo avaliativo seriado dos futuros profissionais (Brasil, 2014). 
A busca contemplou artigos, dissertações, teses, notas, entrevistas, editoriais e livros publicados no período entre 2013 e 2016 em inglês, francês, espanhol e italiano e presentes em uma das três diferentes bases de dados: Biblioteca Virtual em Saúde, portal de periódicos da Capes e Plataforma de Conhecimentos Programa Mais Médicos.

A consulta a elas foi realizada em dois distintos momentos: 14 de abril e 31 de dezembro de 2016. Objetivou-se, ao realizar duas buscas nas bases de dados em períodos distintos, garantir maior fidedignidade dos dados coletados. No dia 14 de abril, foram identificados 143 trabalhos. Destes, 3 o foram excluídos por impertinência temática e 62 por duplicação, sendo selecionados, portanto, 52 trabalhos. A busca do dia 31 de dezembro de 2016 encontrou 383 trabalhos. Destes, 115 foram excluídos por impertinência temática e 140 por duplicações, sendo assim selecionadas 128 produções. A intersecção entre os dois momentos de pesquisa, excluindo-se as repetições (43), gerou um conjunto de 137 trabalhos (Figura 1).

O conjunto de trabalhos selecionados foi submetido à leitura dos resumos, dos quais se extraiu as informações relativas ao tema, aos autores, aos objetivos e aos resultados alcançados. Estas informações foram categorizadas posteriormente segundo: a) ano de publicação; b) tipo de trabalho (artigo, dissertação, tese, outros); c) idioma; d) área do conhecimento, de acordo com a tabela de áreas do conhecimento da Capes, vinculada ao Ministério da Educação (MEC) do Brasil; e) temas e subtemas (provimento, distribuição, formação médica, qualidade da atenção, por exemplo); f) procedência institucional dos pesquisadores, identificada a partir da informação colocada no artigo, dissertação, teses e outros; e g) resultados empíricos apresentados, como a distribuição territorial dos médicos inseridos no programa, a proporção médico/habitante, as características da relação médico/paciente, a satisfação dos profissionais e usuários com o programa etc.

A análise contemplou a sistematização de dados quantitativos, confeccionando-se tabelas e gráficos, bem como a análise do discurso dos autores em relação às características do programa, suas estratégias e seus resultados da implantação.

\section{Figura I - Processo de seleção dos artigos analisados}

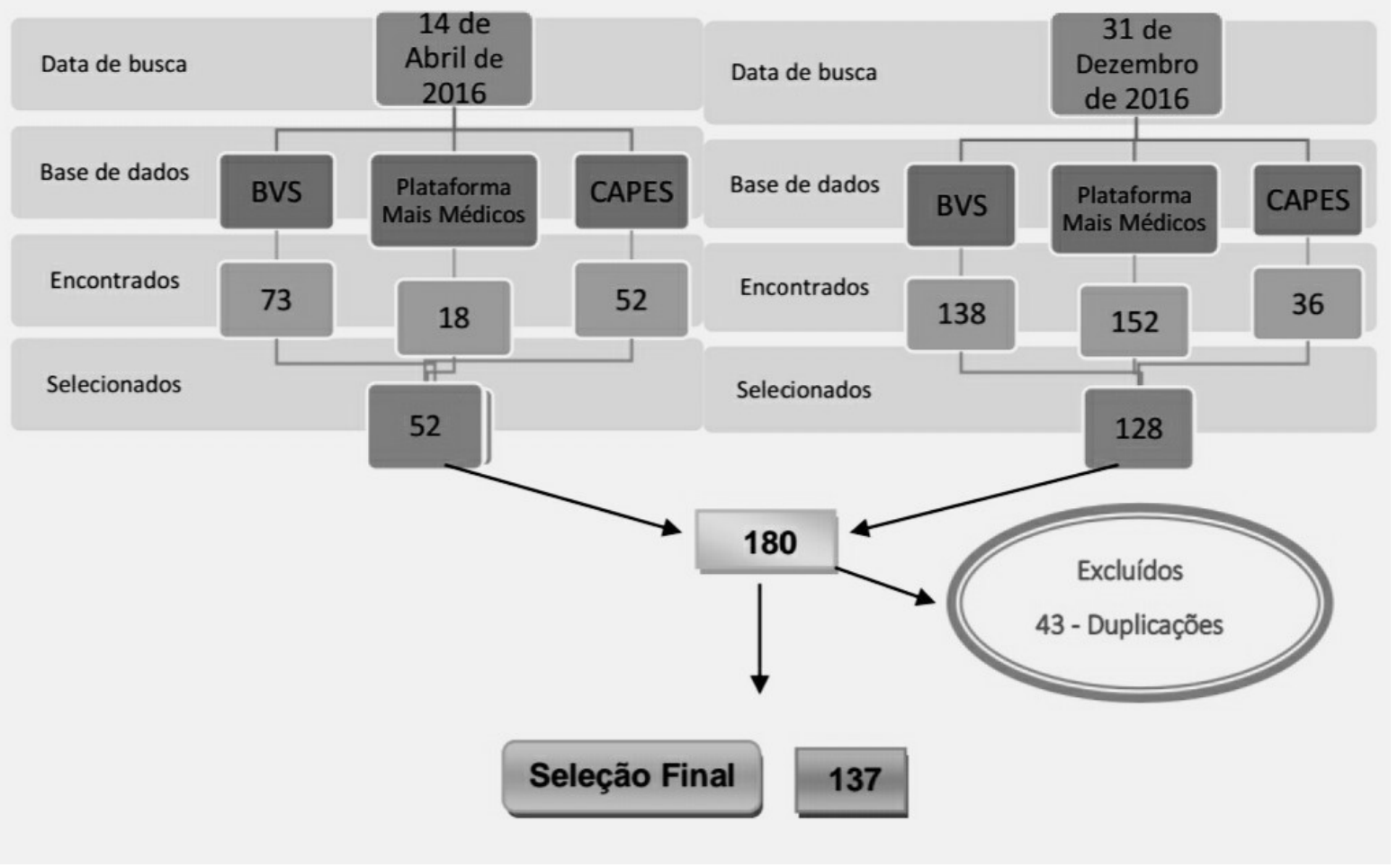




\section{Resultados e discussão}

Os 137 trabalhos selecionados foram analisados em sua integralidade e classificados de acordo com as categorias estabelecidas. Os resultados encontrados são apresentados a seguir.

\section{Distribuição dos estudos/pesquisas segundo tipo de produção e idioma}

Dos 137 trabalhos selecionados, 80 são artigos $(58,4 \%)$. Os demais se distribuem em vários tipos, sendo monografias (8,03 \%) - sejam elas de conclusão de cursos de graduação ou de especialização -, debates $(8,03 \%)$, editoriais $(6,57 \%)$, notas $(5,11 \%)$, dissertações de mestrado $(5,11 \%)$, entrevistas $(2,90 \%)$, teses de doutorado $(2,20 \%)$, livros $(2,20 \%)$ e dossiês $(0,73 \%)$. No que se refere ao idioma dos trabalhos encontrados, a maioria é em português (124 obras), seguida pelas publicações em inglês (oito), espanhol (quatro) e francês (uma).

\section{Distribuição temporal dos estudos}

Os estudos selecionados foram realizados em sua maioria nos anos de 2015 e 2016. No ano de criação do programa, 2013, foram realizados 20 estudos. É importante destacar que em 2016, o mês de junho concentrou a maior parte das publicações, 22 ao total, o que representa 47,82 \% das produções nesse ano (Gráfico 1). Isso provavelmente se deve ao fato de terem sido lançadas várias chamadas públicas de diferentes revistas do campo da saúde coletiva no primeiro semestre de 2016, propostas por entidades como o Comitê Gestor da Rede de Pesquisa em Atenção Primária à Saúde da Associação Brasileira de Saúde Coletiva, a Organização Panamericana de Saúde e o Ministério da Saúde, convocando os pesquisadores da área a realizarem estudos sobre o programa.

\section{Gráfico I - Distribuição temporal dos estudos do PMM}

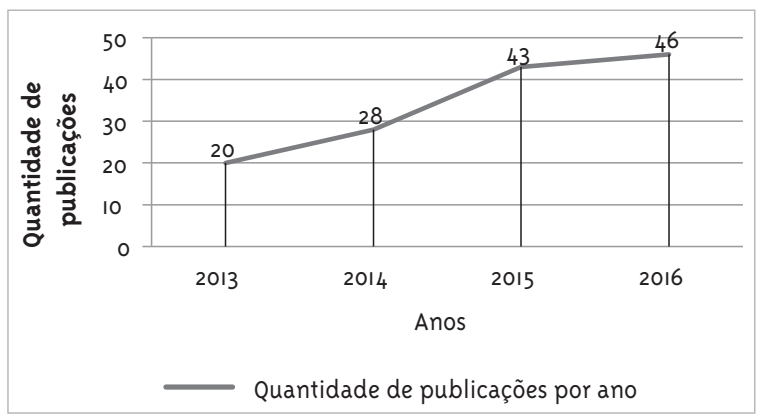

\section{Distribuição segundo a área de conhecimento dos estudos}

Os estudos selecionados são em sua maioria do campo da Saúde Coletiva/Saúde Pública (73\%). Há ainda estudos das áreas de direito, (9\%), administração e comunicação, ambos com 5\% (Gráfico 2).

\section{Gráfico 2 - Distribuição dos estudos sobre o PMM segundo a área do conhecimento}

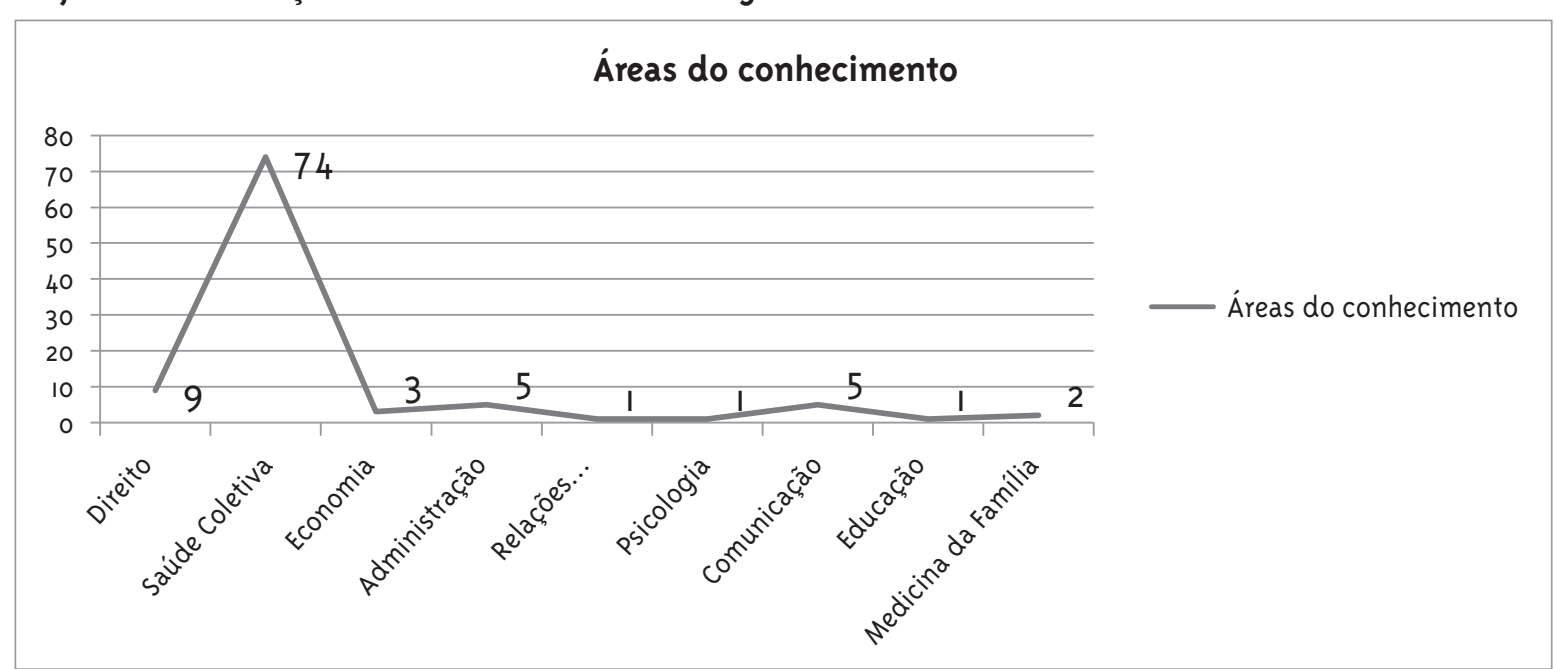




\section{Distribuição institucional e regional dos estudos}

Os estudos selecionados são provenientes de 65 diferentes instituições, sendo 11 internacionais e 54 nacionais. A maioria (54) são universidades, mas também se encontram estudos provenientes do Governo
Federal (dois), de governos estaduais (três), de hospitais (dois), de organizações internacionais (um) e privadas (um), de governos internacionais (um) e de organizações sociais (um). A Universidade de Brasília (UnB) é a instituição com maior quantidade de produções (23), ou seja, 16,78\% dos trabalhos selecionados (Gráfico 3).

\section{Gráfico 3 - Instituições com dois ou mais trabalhos sobre o PMM}

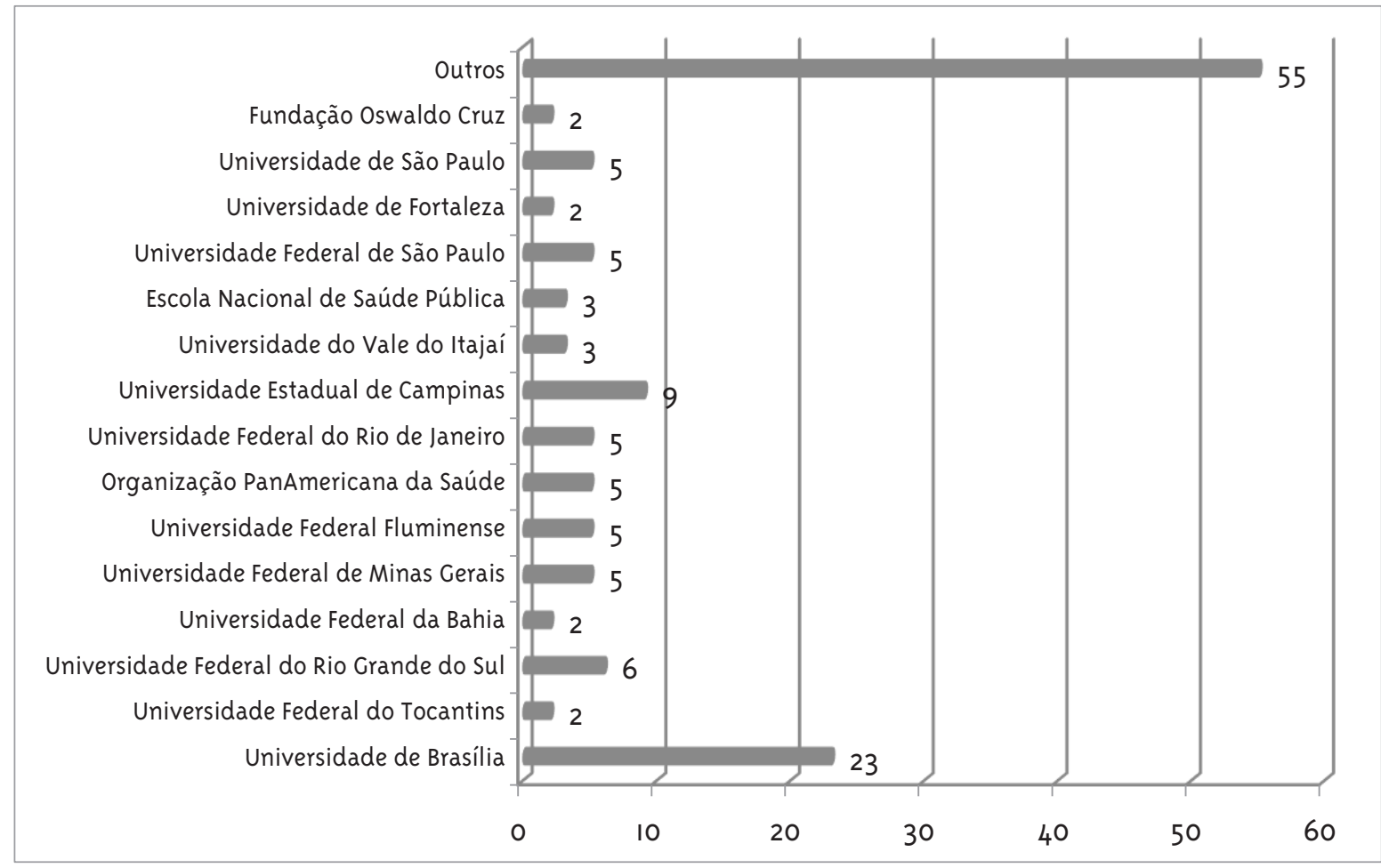

A distribuição territorial dos estudos, por sua vez, revela concentração nas regiões Centro-Oeste, Sudeste e Sul (Figura 2), sendo que a maioria provém do Distrito Federal e de São Paulo, onde se localizam as instituições responsáveis pelas pesquisas. Isso não é exclusividade dos estudos referentes ao PMM, visto que análises anteriores também verificam essa mesma tendência em outras áreas do conhecimento, como a educação, as ciências médicas e biomédicas, a saúde coletiva, a administração, a contabilidade e até mesmo quando se analisa o total da produção científica nacional (Barreto, 2006; Guimarães, 2004; Hocayen-da-Silva, Rossoni; Ferreira Junior, 2008). Algumas hipóteses podem ser levantadas, objetivando explicar tal achado: maior quantidade de grupos de estudos e de pesquisadores, maior quantidade de programas de pós-graduação e maior número de periódicos voltados à publicação de artigos relacionados à saúde coletiva nessas regiões.

No caso específico de Brasília, responsável por 38 das 137 publicações selecionadas, e particularmente a UnB, com 23 publicações, podemos hipoteticamente levantar duas justificativas: localização, uma vez que é justamente no Distrito Federal onde se encontram as principais instituições responsáveis pelo PMM, e formulação de uma rede de pesquisa sobre o PMM, vinculada à UnB, que envolve pesquisadores das Universidades Federais da Bahia, do Pará, de Minas Gerais, de Campina Grande e do Rio Grande do Sul, objetivando analisar os aspectos anteriores à implantação do PMM, sua estrutura, seu processo e seus resultados. 


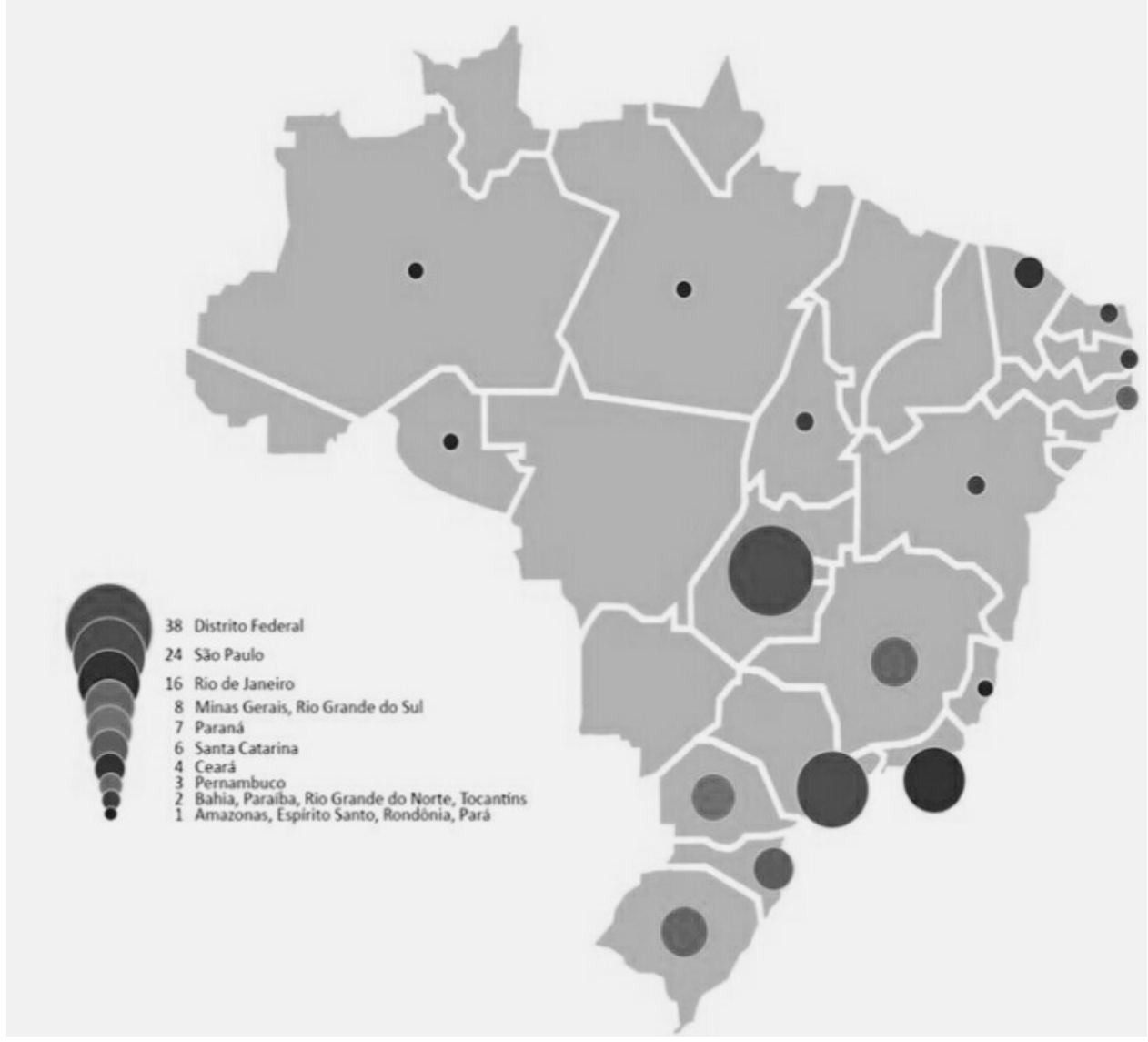

\section{Distribuição segundo tipo de estudo/pesquisa}

O conjunto de artigos, dissertações, mestrados e teses, que totalizam 101 publicações, são em sua maioria pesquisas empíricas (63,36\%), sendo $25,74 \%$ do tipo quantitativa e $37,62 \%$, qualitativa; $25,74 \%$ são ensaios teóricos; revisões de literatura perfazem 5,94\% da amostra e 4,95\%, são relatos de experiência.

\section{Tema e subtemas abordados}

Os mesmos 101 trabalhos foram reagrupados segundo o tema em sete grupos (Tabela 1), sendo que a maioria das publicações tratou da avaliação do programa (34,65\%), seguido das análises políticas sobre o PMM $(31,68 \%)$ e as pesquisas que tratam de provimento e fixação de médicos, (11,89\%). Os demais grupos não ultrapassaram o percentual de 10\%, cabendo destacar que o impacto do PMM sobre o modelo de atenção à saúde foi tema de apenas
$3,96 \%$ dos trabalhos. A seguir, analisamos mais detalhadamente o conteúdo dos artigos incluídos em cada grupo.

Tabela I - Distribuição dos estudos/pesquisas sobre o PMM segundo tema abordado

\begin{tabular}{lcc} 
Tema abordado & $\mathrm{N}$ & $\%$ \\
Avaliação do PMM & 35 & $34,65 \%$ \\
$\begin{array}{l}\text { Análise politica do PMM } \\
\text { Provimento e fixação de } \\
\text { médicos }\end{array}$ & 32 & $31,68 \%$ \\
$\begin{array}{l}\text { O PMM na mídia } \\
\text { Supervisão acadêmica }\end{array}$ & 12 & $11,89 \%$ \\
Modelo de atenção & 07 & $6,93 \%$ \\
Outros & 06 & $5,94 \%$ \\
\hline Total & 04 & $3,96 \%$ \\
\hline
\end{tabular}




\section{Avaliações do PMM}

Os estudos avaliativos sobre o PMM tratam de distintos aspectos do programa, utilizando diferentes abordagens metodológicas (Faria; Paula; Almeida, 2013; Oliveira, F. J., 2014; Pinho, 2014; Piterman et al., 2015; Santos, 2015; Silva; Santos, 2015)

$\mathrm{O}$ aspecto mais importante diz respeito ao impacto do programa sobre o provimento e fixação dos médicos. Vários trabalhos apontam que o PMM foi responsável pelo provimento emergencial de cerca de 14.000 médicos na atenção primária à saúde nos primeiros 10 meses de funcionamento, distribuídos em 3.785 localidades, inclusive em quilombos e aldeias indígenas (Jorge; Martins, 2013; Matte, 2015). Outro impacto apresentado foi o aumento da relação médico por 1.ooo habitantes em diferentes localidades do país e a redução da carência em localidades tradicionalmente excluídas, como é o caso da região Norte do Brasil. Há ainda estudos que discutem o papel da cooperação internacional no provimento desses profissionais e sua importância na implantação do programa (Faria; Paula; Almeida, 2013; Molina et al, 2014; Pinho, 2014; Molina; Tasca; Suarez, 2016)

As principais dificuldades enfrentadas no processo de fixação dos médicos dizem respeito ao fato de determinadas localidades não disporem de condições adequadas de trabalho nem infraestrutura para a realização das atividades primordiais ao exercício médico e sua remuneração, bem como a ausência dos programas de residência em localidades carentes e a ausência de carreira de Estado para os médicos no SUS (Jorge; Martins, 2013, Matte, 2015).

No que se refere ao impacto do programa sobre a educação médica, os trabalhos selecionados apresentam extensa gama de posicionamentos. Alguns sinalizam a necessidade de interiorização e expansão dos cursos de medicina (González; Morales; Ramos, 2015; Matte, 2015; Souza, 2014); outros destacam a importância do estimulo à especialidade em medicina de família e comunidade; outros ainda chamam atenção para a necessidade do favorecimento a novos processos formativos e metodológicos que insiram os estudantes nos estabelecimentos do SUS, considerado um amplo campo de prática e estágios. Ainda nesse grupo, pode-se destacar os artigos que apontam a importância de se analisar e acompanhar os processos formativos nas novas instituições de ensino, criadas a partir da implantação do PMM, e outros, que criticam a quantidade "excessiva" de cursos médicos que vem sendo abertos no território nacional (Amaral; Pêgo-Fernandes; Bibas, 2013; Cruz, 2015; Schanaider, 2014).

Vários estudos destacam que o principal avanço alcançado na formação médica por intermédio do PMM foi a instituição das novas DCN para os cursos de graduação em medicina, em 2014, com a exigência de uma carga horária mínima em atenção básica e nos serviços de urgência/emergência. Destacam-se ainda os impactos nos programas de residência médica, com a ampliação da quantidade de vagas e regulação da sua distribuição, e no modelo ideológico centrado na medicina da família e da comunidade, visto que esta passa a ser a entrada para a maioria das especializações no país (Silva Junior; Andrade, 2016).

No que se refere à relação médico-paciente, há trabalhos que a investigam usando a análise do discurso de diferentes sujeitos, como profissionais, usuários e gestores. De modo geral, esses trabalhos destacam a satisfação dos usuários com os serviços prestados, suas percepções em relação aos profissionais, suas expectativas, as principais dificuldades no diálogo entre profissional e paciente e suas avaliações, salientando as dificuldades de acesso aos serviços anteriores ao programa e as condições atuais das unidades de saúde (Comes et al., 2016; Costa et al., 2015; Melo; Baião; Costa, 2016; Rodrigues, 2015). Tais estudos demonstram que os pacientes consideram o PMM uma iniciativa relevante, sendo que boa parte deles afirma ter ocorrido uma melhoria no atendimento, destacando que os profissionais, principalmente os estrangeiros, "tem mais atenção, interesse, interação, paciência, dão mais espaço, olham, ouvem e conversam com o paciente" (Brito, 2014, p. 69). Chama atenção o fato de que alguns trabalhos destacam que os usuários percebem o atendimento realizado pelos profissionais cubanos como superior ao desenvolvido pelos seus pares brasileiros, destacando como principal diferencial o olhar, a escuta, a atenção e o respeito (Comes et al., 2016; Melo; Baião; Costa, 2016; Silva et al., 2016).

A infraestrutura das unidades de saúde também é objeto de alguns estudos, a exemplo 
dos que analisam a efetividade dos investimentos realizados e seus impactos nos indicadores de saúde, destacando o aumento da produtividade das equipes de saúde da família, a redução de iniquidades, a ampliação do acesso e da cobertura da assistência à saúde, a redução do tempo de espera e o aumento da resolutividade da demanda (Giovanella et al., 2016; Girardi et al., 2016b; Soares; Machado; Alves, 2016).

\section{Análise política do PMM}

Da amostra selecionada, 31,68\% apresenta a análise da conjuntura em que foi lançado o PMM, destacando como possíveis causas da criação do programa: as demandas sociais (Angotti Neto, 2013; Magno; Paim, 2015), a necessidade de provimento de médicos (Girardi et al., 2016a; Rodrigues, 2015), a melhoria da infraestrutura das unidades de saúde (Lima et al., 2015; Macinko; Harris, 2015) ou a necessidade por mudanças na formação médica (Costa et al., 2015).

Os estudos que analisam o processo de elaboração do programa, por sua vez, utilizam vários referenciais de análise de políticas e diferentes metodologias, que vão desde revisão documental (Kemper; Mendonça; Sousa, 2016; Oliveira et al., 2015) e revisão histórica (Mendes et al., 2015) até pesquisa empírica (Macedo et al., 2015) com entrevistas realizadas com os atores que participaram do processo de elaboração do programa. Chama atenção, inclusive, o uso do referencial do ciclo da política proposto por John W. Kingdon (Couto, 2015).

Nessa perspectiva, alguns estudos classificam o processo de formulação do PMM como um modelo top down de políticas públicas (Kamikawa; Motta, 2014), uma vez que as decisões sobre a criação do programa foram tomadas de cima para baixo. Outros o analisam como uma disposição "populista”, na medida em que enfatizam o fato de sua elaboração ter sido uma resposta às pressões populares que explodiram nas jornadas de junho de 2013 (Alessio, 2015; Ribeiro, 2015). Nesse grupo, há ainda alguns estudos que o consideram como uma política intersetorial, visto que sua elaboração envolveu diferentes atores, inseridos em diversos órgãos governamentais federais (Ministério da Saúde, MEC e Ministério do Planejamento), apresentando, portanto, discursos, posicionamentos e objetivos desses diferentes atores sociais e entidades envolvidas (Lotta, 2016; Silva, 2016).

Vários estudos apresentam análise jurídica referente à inserção de médicos estrangeiros no programa (Matte, 2015; Santos, 2015; Silva; Santos, 2015). Outros comparam o desenvolvimento do programa no Brasil com outras estratégias, implementadas em diferentes países - por exemplo, a comparação da política de provimento médico brasileira com a que é desenvolvida na Austrália e nos Estados Unidos (Piterman et al., 2015).

\section{O impacto do PMM sobre o modelo de atenção}

Os estudos analisados demonstram que, embora recente, o PMM tem ampliado as ações da Estratégia de Saúde da Família, permitindo maior integração entre os diferentes profissionais e potencializando suas atividades. Assim, as ações desenvolvidas pelo PMM e a atenção em saúde concedida pelos profissionais aos pacientes abordam diferentes dimensões do cuidado: a promoção da saúde, a prevenção de doenças e agravos, o diagnóstico e o tratamento de doenças, que agora extrapolam o uso de medicamentos e valorizam aspectos culturais e tradicionais da população (Gomes, 2014; Molina et al., 2014; Santos et al., 2015)

Alguns estudos destacam que as mudanças na produção de ações e serviços podem ser explicadas por dois fatores principais: a vinda de profissionais estrangeiros, principalmente cubanos, que possuem prática médica mais promocional e preventiva e que tendem a se afastar do modelo hospitalocêntrico, valorizando os aspectos culturais da população e garantido atenção mais integral (Vargas; Campos; Vargas, 2016), e o fato do PMM ter concedido importância significativa à Atenção Básica em Saúde, o que serviu de base para a reorientação da formação médica e dos programas de residência (Bertão, 2015; Comes et al., 2016; Mendonça, Diercks, Kopittke, 2016).

Apesar desses avanços é necessário destacar que alguns estudos enfatizam que o caráter provisório da inserção dos médicos do programa nas unidades básicas de saúde (UBS), pode ser um empecilho à vinculação e à criação de relações da população com as unidades de saúde, ainda que os 
usuários relatem maior aproximação com as UBS em decorrência da disponibilidade constante de médicos, da maior facilidade no agendamento das consultas, do aumento de visitas domiciliares e menor tempo de espera para as consultas de retorno (Pinto et al., 2014).

Constata-se, portanto, que o PMM se insere no conjunto de iniciativas que podem contribuir para a reorientação do modelo de atenção no sistema de saúde, porquanto as práticas profissionais realizadas no âmbito do programa articulam algumas das propostas que vem sendo discutidas e implementadas visando a integralidade da atenção, especialmente a oferta organizada de serviços por meio do acolhimento, as ações programáticas de saúde e o fortalecimento da Estratégia de Saúde da Família, possibilitando a articulação de ações de educação sanitária, vigilância epidemiológica, assistência a grupos populacionais prioritários e a reorganização da atenção à demanda espontânea.

\section{Supervisão acadêmica promovida pelo PMM}

Estudos recentes direcionam-se para a análise da supervisão e tutoria aos bolsistas do PMM, desenvolvida por médicos com experiência em atenção básica, destacando que esse processo deve ser embasado nas diretrizes pedagógicas da Educação Permanente e objetivar o desenvolvimento de competências necessárias para se trabalhar na Estratégia de Saúde da Família. Assim, cabe ao supervisor fortalecer a educação permanente, a interação ensino-serviço e a atenção básica, bem como participar e orientar sobre o processo formativo dos bolsistas nas redes de atenção à saúde e garantir a articulação dos eixos educacionais do PMM (Engstrom et al., 2016; Lima et al., 2016).

As metodologias utilizadas no processo de supervisão são os principais objetos de estudo, defendendo-se a utilização daquelas participativas, que envolvam a problematização e respeitem o conhecimento prévio dos envolvidos no processo formativo. Outro aspecto analisado é a abrangência dessa supervisão: alguns estudos afirmam que ela se restringe apenas ao profissional médico, o que não influenciaria o modo de trabalho da equipe como um todo, já que há outros profissionais de saúde envolvidos no processo de trabalho, minimizando, assim, o impacto das possíveis ações que poderiam vir a serem desenvolvidas; outras pesquisas concluem que o processo de educação, além do suporte individual ao médico do programa, contempla a observação do processo de trabalho da equipe na qual o médico está inserido em sua totalidade, permitindo assim melhor interação entre os profissionais e as equipes municipais (Castro, 2015; Rodrigues; Quaresma; Monteiro, 2015)

Os principais desafios elencados durante a supervisão são: presença de profissionais jovens, com média de dois a três anos de vivência profissional; formação dos bolsistas em instituições de currículo com metodologia tradicional, voltandose principalmente para o assistencialismo (tal aspecto é agravado principalmente em meio aos profissionais brasileiros); dificuldade em propiciar o acordo de interesses entre gestores e profissionais da equipe de saúde (Castro, 2015; Engstrom et al., 2016 ; Lima et al., 2016; Rodrigues; Quaresma; Monteiro, 2015).

\section{o PMM na mídia}

A reação de diferentes categorias e atores sociais é analisada por meio dos seus discursos em diferentes mídias, sejam elas nos jornais impressos de comunicação de massa, a exemplo da Folha de S.Paulo (Levy; Santos, 2015), do Correio Braziliense (Santos, Costa; Girardi, 2015), ou da revista Veja (Pereira; Silva; Santos, 2015); jornais televisivos, como o Bom Dia, Brasil (Vanzella, 2014), da rede Globo de televisão; sites da internet, como o Pragmatismo Político e o Portal Médico (Alencar et al., 2016; Mota; Barros, 2016); e redes sociais, como o Facebook (Silva et al., 2016).

Os estudos destacam principalmente as percepções das entidades representativas das categorias médicas e os posicionamentos pessoais de alguns médicos brasileiros em relação ao programa. As críticas se concentram, principalmente, na abertura de novas vagas nos cursos de medicina em localidades sem infraestrura adequada e com escassez de corpo docente tecnicamente qualificado, que poderiam propiciar a precarização da formação médica. No que se refere à vinda de médicos estrangeiros, 
vários artigos que analisam as publicações da mídia apontam a reação negativa das entidades e dos médicos entrevistados com relação à não exigência de revalidação do diploma, que afirmaria a capacidade dos profissionais e a qualidade da assistência prestada, ademais salientam que haveria uma persistências de ações voltadas para aspectos quantitativos, que negligenciariam politicas voltadas para a melhoria da qualidade nas unidades de saúde e a possibilidade da elaboração de um plano de carreira estatal para os médicos (Alencar et al., 2016; Mota; Barros, 2016).

Como se pode perceber, os posicionamentos políticos acerca do programa evidenciam a existência de uma polêmica, sendo necessário destacar que as visões apresentadas pelas entidades médicas, como o Conselho Federal de Medicina (CFM) e a Associação Médica Brasileira (ABM), são distintas dos posicionamentos de outras instituições, como o Centro Brasileiro de Estudo de Saúde (Cebes) e a Associação Brasileira de Saúde Coletiva (Abrasco). Os trabalhos indicam que essas entidades apresentam pontos de vista antagônicos. Se por um lado os atores médicos se expressam contrariamente ao PMM, os sujeitos do campo da saúde coletiva elogiam a proposta e a apoiam (Alessio, 2015; Silva Junior; Andrade, 2016; Silva Junior et al., 2016).

Finalmente, cabe destacar que algumas investigações apresentam como resultado a relação entre o discurso negativo acerca do PMM e as críticas ao governo do Partido dos Trabalhadores (PT), particularmente na figura da ex-presidente Dilma Rousseff, o que demonstra o grau de politização conferido ao PMM, que se tornou, no período estudado, um dos principais alvos das forças contrárias às políticas sociais implementadas no período 2003-2013 - expressas, nesse caso específico, pela intensificação de uma visão corporativa, que se apresentou claramente nos editoriais das publicações do CFM (Alessio, 2015; Silva Junior; Andrade, 2016; Silva Junior et al., 2016).

\section{Considerações finais}

Com base na revisão de literatura apresentada e discutida nos itens anteriores, pode-se apresentar as seguintes conclusões:
1. Apesar de todas as discussões e críticas em torno da criação e implementação do PMM, principalmente por parte das entidades médicas, o programa representa um grande avanço em termos de política pública que integra, em uma única proposta, elementos voltados ao provimento emergencial de médicos, a educação médica e ao desenvolvimento da infraestrutura das unidades de saúde no âmbito do SUS.

2. Os resultados iniciais da avaliação do PMM indicam que o programa tem reduzido iniquidades em saúde, aumentado a proporção médico/habitante e melhorado a qualidade da relação médico-paciente, propiciando atendimentos mais humanizados, ao mesmo tempo em que tem favorecido a integração das práticas dos diferentes profissionais das equipes de saúde e aumentado a efetividade das ações nas UBS.

3. No que diz respeito ao impacto do PMM sobre a educação médica, é importante destacar que ele responde às demandas formuladas por diversos setores da sociedade e estudiosos desse campo, que solicitavam uma formação mais ampla, capaz de extrapolar o âmbito hospitalar e envolver os alunos por meio de novas metodologias problematizadoras, em diferentes cenários de prática/realidades de atenção. Assim, o PMM avança significativamente ao propor mudanças nas DCN e determinar a criação de uma avaliação seriada dos futuros profissionais.

4. Por último, cabe ressaltar que as obras referentes ao PMM começaram a ser publicadas recentemente, ainda que tenhase observado uma tendência recente ao crescimento do volume delas. O estudo realizado mostra-se relevante, visto que foi capaz de elencar aspectos da fase inicial do programa, bem como apresentar sistematicamente os resultados das avaliações iniciais e apontar temas dos demais estudos a serem realizados. 


\section{Referências}

ALENCAR, A. P. A. et al. Impacto do programa mais médicos na atenção básica de um município do sertão central nordestino. Gestão \& Sociedade, Belo Horizonte, v. 10, n. 26, p. 1290-1301, 2016.

ALESSIO, M. M. Análise da implantação do programa mais médicos. 2015. 90 f. Dissertação (Mestrado em Saúde Coletiva) - Universidade de Brasília, Brasília, 2015.

AMARAL, J. L. G.; PÊGO-FERNANDES, P. M.; BIBAS, B. J. Mais médicos: melhor atenção à saúde da população? Diagnóstico e Tratamento, São Paulo, v. 17, n. 1, p. 3-4, 2012.

ANGOTTI NETO, H. Políticas de interiorização do médico brasileiro. Ibérica, Juiz de Fora, v. 7, n. 21, p. 41-56, 2013.

BARRETO, M. L. Crescimento e tendência da produção científica em epidemiologia no Brasil. Revista de Saúde Pública, São Paulo, v. 4o, n. esp., p. 79-85, 2006.

BERTÃO, I. R. A atuação de um médico do Programa Mais Médicos para o Brasil e a mudança processo de trabalho da equipe de Estratégia de Saúde da Família. Tempus: Actas de Saúde Coletiva, Brasília, v. 9, n. 4, p. 217-224, 2015. BRASIL. Biblioteca: Presidência da República. o8-07-2013 - Discurso da Presidenta da República, Dilma Rousseff, durante cerimônia de lançamento do Pacto Nacional pela Saúde - Mais Hospitais e Unidades de Saúde, Mais Médicos e Mais Formação. Brasília, DF, 8 jul. 2013a. Disponível em: <https://bit.ly/2mz7ifT >. Acesso em: 10 jan. 2017.

BRASIL. Lei ${ }^{0}{ }^{12.871}$, de 22 de outubro de 2013. Institui o Programa Mais Médicos, altera as Leis $n^{0}$ 8.745, de 9 de dezembro de 1993, e n 6.932, de 7 de julho de 1981, e dá outras providências. Diário Oficial da União, Brasília, DF, 22 out. 2013b. Disponível em: <https://bit.ly/2JJV1OZ>. Acesso em: 22 jul. 2018.

BRASIL. Resolução CES n ${ }^{0}$ 3, de 20 de junho de 2014. Institui Diretrizes Curriculares Nacionais do Curso de Graduação em Medicina e dá outras providências. Diário Oficial da União, Brasília,
DF, 23 jun. 2014. Disponível em: <https://bit. ly/2k7LtEn>. Acesso em: 22 jul. 2018.

BRASIL. Ministério da Saúde. Programa mais médicos - dois anos: mais saúde para os brasileiros. Brasília, DF, 2015.

BRINGEL, B.; PLEYERS, G. Les mobilisations de juin 2013. Paris: Éditions de la Maison des Sciences de L'homme, 2013.

BRITO, L. M. Programa Mais Médicos: Satisfação dos usuários da atenção básicas de saúde de Ceilândia/DF quanto ao atendimento médico. 2014. 93 f. Monografia (Bacharelado em Saúde Coletiva) - Universidade de Brasília, Ceilândia, 2014.

BUENO, I. M.; SILVA, M. B. O. Constituinte e lutas populares: o materialismo da Constituição e as lutas pela Constituinte Exclusiva. Culturas Jurídicas, Niterói, v. 1, n. 2, p. 243-266, 2015.

CASTRO, T. F. Reflexões sobre a prática de supervisão no Programa de Valorização do Profissional da Atenção Básica (PROVAB) e no Programa Mais Médicos: resumos de teses e dissertações. Tempus: Actas de Saúde Coletiva, Brasília, v. 9, n. 4, p. 237-238, 2015.

COMES, Y. et al. Avaliação da satisfação dos usuários e da responsividade dos serviços em municípios inscritos no Programa Mais Médicos. Ciência \& Saúde Coletiva, Rio de Janeiro, v. 21, n. 9, p. 2749-2759, 2016.

COSTA, A. M. et al. Mais (e melhores) médicos. Tempus: Actas de Saúde Coletiva, Brasília, v. 9, n. 4 , p. 175-181, 2015 .

COUTO, M. P. O Programa Mais Médicos: a formulação de uma nova política pública de saúde no Brasil. 2015. 198 f. Dissertação (Mestrado em Políticas Públicas) - Universidade Federal do Paraná, Curitiba: 2015.

CRUZ, M. A. C. A integralidade nas práticas de saúde após implantação do Programa Mais Médicos: a experiência da Unidade Básica de Saúde Samambaia - Juatuba-MG. 2015. 175 f. Dissertação (Mestrado em Saúde Coletiva) - Universidade Estadual de Campinas, Campinas, 2015. 
ENGSTROM, E. M. et al. O supervisor e as estratégias educacionais dos encontros locorregionais no Programa Mais Médicos do Brasil: reflexões acerca de concepções e práticas. Tempus: Actas de Saúde Coletiva, Brasília, v. 10, n, 1, p. 241-252, 2016.

FARIA, M. A.; PAULA, D. M. P.; ALMEIDA; J. D. L. Cooperações técnicas bilaterais de saúde entre Brasil e organismos internacionais. Cadernos Ibero-Americanos de Direito Sanitário, Brasília, v. 2, n. 2, p. 960-97o, 2013.

GIOVANELLA, L. et al. A provisão emergencial de médicos pelo Programa Mais Médicos e a qualidade da estrutura das unidades básicas de saúde. Ciência \& Saúde Coletiva, Rio de Janeiro, v. 21, n. 9, p. 2697-2708, 2016.

GIRARDI, S. N. et al. Avaliação do escopo de prática de médicos participantes do Programa Mais Médicos e fatores associados. Ciência \& Saúde Coletiva, Rio de Janeiro, v. 21, n. 9, p. 27392748, 2016a.

GIRARDI, S. N. et al. Impacto do Programa Mais Médicos na redução da escassez de médicos em atenção primária à saúde. Ciência $\&$ Saúde Coletiva, Rio de Janeiro, v. 21, n. 9, p. 2675-2684, 2016b.

GONZÁLEZ, J. E. C; MORALES, E. C. L; RAMOS, A. D. Proyecto "Mais Médicos para Brasil" nueva modalidad de colaboración cubana en el exterior. Edumecentro, Santa Clara, v. 7, n. 1, p. 171-177, 2015.

GOMES, R. M. Redes de Atenção à Saúde do SUS: 25 anos de uma contradição fundamental entre a organização necessária e a organização atual. Saúde em Debate, Rio de Janeiro, v. 38, n.103, p. 938-952, 2014.

GUIMARÃES, J. A. A pesquisa médica e biomédica no Brasil: comparações com o desempenho científico brasileiro e mundial. Ciência \& Saúde Coletiva, Rio de Janeiro, v. 9, n. 2, p. 303-327, 2004.

HOCAYEN-DA-SILVA, A. J.; ROSSONI, L.; FERREIRA JUNIOR, I. Administração pública e gestão social: a produção científica brasileira entre 2000 e 2005. Revista de Administração Pública, Rio de Janeiro, v. 42, n. 4, p. 655-68o, 2008.
JORGE, D.; MARTINS, F. Estudo jurídico do Programa Mais Médicos. Acta Jus, Maringá, v. 1, n. 1, p. 24-34, 2013.

KAMIKAWA, G. K.; MOTTA, I. D. Direito à saúde e estudo da política pública do Programa Mais Médicos. Revista Jurídica Cesumar: Mestrado, Maringá, v. 14, n. 2, p. 341-367, 2014.

KEMPER, E. S.; MENDONÇA, A. V. M; SOUSA, M. F. Programa Mais Médicos: panorama da produção científica. Ciência \& Saúde Coletiva, Rio de Janeiro, v. 21, n. 9, p. 2785-2796, 2016.

LEVY, D. R.; SANTOS, C. E. G. O Programa Mais Médicos e os impactos na saúde da população brasileira. Revista Brasileira de Previdência: Atuária e Contabilidade e Direito Previdenciário, Osasco, n. 4, 2015.

LIMA, R. C. G. S. et al. Programa Mais Médicos no estado de Santa Catarina: breve relato de experiências iniciais. Tempus: Actas de Saúde Coletiva, Brasília, v. 9, n. 4, p. 67-80, 2015.

LIMA, R. C. G. S. et al. Tutoria acadêmica do Projeto Mais Médicos para o Brasil em Santa Catarina: perspectiva ético-política. Ciência \& Saúde Coletiva, Rio de Janeiro, v. 21, n. 9, p. 27972805, 2016.

LOTTA, G. S. Análise do Programa Mais Médicos à luz dos arranjos institucionais: intersetorialidade, relações federativas, participação social e territorialidade. Ciência \& Saúde Coletiva, Rio de Janeiro, v. 21, n. 9, p. 2761-2772, 2016.

MACEDO, A. S.; ALCANTARA, V. C.; ANDRADE, L. F. S.; FERREIRA, P. A. O papel dos atores na formulação e implementação de políticas públicas: dinâmicas, conflitos e interesses no Programa Mais Médicos. Cadernos EBAPE.BR, Rio de Janeiro, v. 14, n., p. 593-618, 2016.

MACINKO, J.; HARRIS, M. J. Brazil's family health strategy delivering community-based primary care in a universal health system. The New England Journal of Medicine, Boston, v. 372, n. 23, p. 2177-2181, 2015.

MAGNO, L. D.; PAIM, J. S. Dos clamores das ruas aos rumores no Congresso: uma análise da conjuntura recente da saúde no Brasil. Reciis: Revista 
Eletrônica de Comunicação, Informação e Inovação em Saúde, Rio de Janeiro, v. 9, n. 4, p. 1-14, 2015.

MATTE, N. A. A justiça do trabalho e o discurso normativo: a violação das garantias trabalhistas no Programa Mais Médicos para o Brasil (lei 12.871/2013). 2015. 58 f. Monografia (Bacharelado em Direito) - Centro Universitário de Brasília, Brasília, 2015.

MENDES, L. Z. et al. Programa Mais Médicos: retrospectiva de seu contexto histórico e implantação no município de São Borja/RS. In: SALÃO INTERNACIONAL DE ENSINO, PESQUISA E EXTENSÃO, 7., 2015, São Borja. Anais... Bagé: Universidade Federal do Pampa, 2015.

MELO, C. F.; BAIÃO, D. C.; COSTA, M. C. A percepção dos usuários cearenses sobre o Programa Mais Médicos. Gestão \& Sociedade, Belo Horizonte, v. 10, n. 26, p. 1302-1312, 2016.

MENDONÇA, C. S.; DIERCKS, M. S.; KOPITTKE, L. $\mathrm{O}$ fortalecimento da atenção primária à saúde nos municípios da região metropolitana de Porto Alegre, Brasil, após a inserção no Programa Mais Médicos: uma comparação intermunicipal. Ciência \& Saúde Coletiva, Rio de Janeiro, v. 21, n. 9, p. 2871-2878, 2016.

MOLINA, J.; TASCA, R.; SUAREZ, J. Monitoramento e avaliação do Projeto de Cooperação da OPAS/ OMS com o Programa Mais Médicos: reflexões a meio caminho. Ciência \& Saúde Coletiva, Rio de Janeiro, v. 21, n. 9, p. 2925-2933, 2016.

MOLINA, J. et al. O programa Mais Médicos e as redes de atenção à saúde no Brasil. Saúde para Debate, Rio de Janeiro, n. 52, p. 190-201, 2014.

MOTA, R. G.; BARROS, N. F. O Programa Mais Médicos no estado de Mato Grosso, Brasil: uma análise de implementação. Ciência \& Saúde Coletiva, Rio de Janeiro, v. 21, n. 9, p. 2879-2888, 2016.

OLIVEIRA, F. J. Contratação de estrangeiros sob a lei federal 12.871/2013 (Projeto Mais Médicos para o Brasil). Revista de Direito Administrativo Contemporâneo, São Paulo, v. 2, n. 9, p. 59-73, 2014.

OLIVEIRA, F. P. et al. Mais médicos: um programa brasileiro em uma perspectiva internacional.
Interface: Comunicação, Saúde, Educação, Botucatu, v. 19, n.54, p. 623-635, 2015.

OPS - ORGANIZACIÓN PANAMERICANA DE LA SALUD. Investigación cualitativa en enfermería: metodología y didáctica. Washington, DC, 2013.

PEREIRA, L. L; SILVA, H. P.; SANTOS, L. M. P. Projeto Mais Médicos para o Brasil: estudo de caso em comunidades quilombolas. Revista da $A B P N$, Goiânia, v. 7, n. 16, p. 28-51, 2015.

PINHO, M. O. A intermediação de mão-de-obra na administração pública por meio de organismos internacionais: problemas quanto à efetividade das normas de proteção trabalhista. 2014. 82 f. Monografia (Bacharelado em Direito) Universidade de Brasília, Brasília, 2014

PINTO, H. A. et al. O Programa Mais Médicos e o fortalecimento da atenção básica. Divulgação em Saúde para Debate, Londrina, n. 51, p. 105-120, 2014.

PITERMAN, L. et al. International primary care snapshots: Australia and Brazil. British Journal of General Practice, London, v. 65, n. 633, p. 198-199, 2015.

RIBEIRO, R. C. Programa Mais Médicos - um equívoco conceitual. Ciência \& Saúde Coletiva, Rio de Janeiro, v. 2O, n. 2, p. 421-424, 2015.

RODRIGUES, K. M. A. O Programa Mais Médicos do Governo Federal: um aporte pedagógico na saúde para a construção da cidadania. Tempus: Actas de Saúde Coletiva, Brasília, v. 9, n. 4, p. 35-47, 2015.

RODRIGUES, C. C. P.; QUARESMA, M. S. M.; MONTEIRO, R. C. Educação em saúde no Programa Mais Médicos para o Brasil: o papel do supervisor no processo educacional. Tempus: Actas de Saúde Coletiva, Brasília, v. 9, n. 4, p. 151-158, 2015.

SANTOS, M. C. F. Programa Mais Médicos: estrutura do Programa Mais Médicos e implementação do Projeto Mais Médicos para o Brasil. 2015. 56 f. Monografia (Especialização em Políticas Públicas) - Universidade Federal de Minas Gerais, Belo Horizonte; 2015.

SANTOS, L. M. P.; COSTA, A. M.; GIRARDI, S. N. Programa Mais Médicos: uma ação efetiva para reduzir iniquidades em saúde. Ciência \& Saúde Coletiva, Rio de Janeiro, v. 20, n. 11, p. 3547-3552, 2015. 
SANTOS, J. B. F. et al. A representação social dos cearenses sobre o Programa Mais Médicos. In: ENCONTRO DE PÓS-GRADUAÇÃO E PESQUISA, 14., 2014, Fortaleza. Anais... Fortaleza: Universidade de Fortaleza, 2014.

SCHANAIDER, A. Mais ou menos médicos! Revista do Colégio Brasileiro de Cirurgiões, Rio de Janeiro. v. 41, n. 5, p. 303-304, 2014.

SILVA, I. E. P. "Tem hora que a gente se pergunta por que é que não se junta tudo numa coisa só?”: Programa Mais Médicos para o Brasil - caminhos da intersetorialidade. 2016. 43 f. Monografia (Especialização em Saúde Coletiva e Educação na Saúde) - Universidade Federal do Rio Grande do Sul, 2016.

SILVA, S. M; SANTOS, L. M. P. Estudo das ações diretas de inconstitucionalidade do Programa Mais Médicos. Cadernos Ibero-Americanos de Direito Sanitário, Brasília, v. 4, n. 2, p. 68-82, 2015.

SILVA, T. R. B. et al. Percepção de usuários sobre o Programa Mais Médicos no município de Mossoró, Brasil. Ciência \& Saúde Coletiva, Rio de Janeiro, v. 21, n. 9, p. 2861-2869, 2016.

SILVA, B. P. et al. Ampliação do acesso à saúde na região mais vulnerável do estado de São Paulo, Brasil: reflexo do Programa Mais Médicos? Ciência \& Saúde Coletiva, Rio de Janeiro, v. 21, n. 9, p. 2899-29o6, 2016.
SILVA JUNIOR, A. G.; ANDRADE, H. S. Formação médica no Programa Mais Médicos: alguns riscos. Ciência \& Saúde Coletiva, Rio de Janeiro, v. 21, n. 9, p. 2670-2671, 2016.

SILVA JUNIOR, A. G. et al. Oferta de vagas de graduação e residência médicas no estado do Rio de Janeiro a partir do Programa Mais Médicos (2013-2015). Gestão \& Sociedade, Belo Horizonte, v. 10, n. 26, p. 1347-1359, 2016.

SOARES, J. J.; MACHADO, M. H.; ALVES, C. B. O Programa Mais Médicos, a infraestrutura das Unidades Básicas de Saúde e o Índice de Desenvolvimento Humano Municipal. Ciência \& Saúde Coletiva, Rio de Janeiro, v. 21, n. 9, p. 27092718, 2016.

SOARES, C. L. M. et al. Análise do posicionamento das entidades médicas - 2015-2016. Saúde em Debate, Rio de Janeiro, v. 41, n. esp. 3, p. 74-86, 2017.

SOUZA, L. Representação do "Programa Mais Médicos" pelo site Pragmatismo Político e pelo portal do Conselho Federal de Medicina. 2014. 144 f. Dissertação (Mestrado em Linguística) Universidade de Brasília, Brasília, 2014.

VARGAS, A. F. M.; CAMPOS, M. M.; VARGAS, D. S. O risco dos extremos: uma análise da implantação do Programa Mais Médicos em um contexto de volatilidade orçamentária. Gestão \& Sociedade, Belo Horizonte, v.10, n. 26, p. 1313-1326, 2016.

\section{Contribuição dos autores}

Rios foi responsável pela concepção e redação do manuscrito, coleta de dados, formatação das imagens, adequação às normas da revista, submissão e reformulação. Teixeira orientou a pesquisa inicial que deu origem à ideia do presente artigo e, participou da revisão crítica e revisão final.

Recebido: 19/11/2017

Reapresentado: 02/05/2018

Aprovado: 15/05/2018 